\title{
Numerical Investigation on a Prototype Centrifugal Pump Subjected to Fluctuating Rotational Speed
}

\author{
Yu-Liang Zhang, ${ }^{1}$ Jun-Jian Xiao, ${ }^{1}$ Yan-Jun Zhao, ${ }^{2}$ and Ying-Yu Ji ${ }^{1}$ \\ ${ }^{1}$ College of Mechanical Engineering, Quzhou University, Quzhou 324000, China \\ ${ }^{2}$ Quzhou College of Technology, Quzhou 324000, China \\ Correspondence should be addressed to Yu-Liang Zhang; zhang002@sina.com
}

Received 26 February 2014; Accepted 30 April 2014; Published 26 June 2014

Academic Editor: Hua-Shu Dou

Copyright (c) $2014 \mathrm{Yu}$-Liang Zhang et al. This is an open access article distributed under the Creative Commons Attribution License, which permits unrestricted use, distribution, and reproduction in any medium, provided the original work is properly cited.

\begin{abstract}
The rotational speed of pumps often encounters fluctuation in engineering for some reasons. In this paper, in order to study the transient response characteristic of a prototype centrifugal pump subjected to fluctuating rotational speed, a closed-loop pipe system including the pump is built to accomplish unsteady flow calculations in which the boundary conditions at the inlet and the outlet of the pump are not required to be set. The external performance results show that the head's responsiveness to the fluctuating rotational speed is very good, while the flow rate's responsiveness is slightly delayed. The variation tendencies of the static pressures at the inlet and the outlet of the pump are almost completely opposite, wherein the variation tendency of the static pressure at the outlet is identical with that of the rotational speed. The intensity of the turbulence energy in each impeller channel is relatively uniform in the transient flow calculations, while, in the quasi-steady flow calculation, it becomes weaker in a channel closed to the volute tongue. The nondimensional flow rate and head coefficients are dependent on the rotational speed, and their variation tendencies are opposite to that of the fluctuating rotational speed as a whole.
\end{abstract}

\section{Introduction}

As is known to all, vane pumps usually operate at stable working points; namely, the rotational speed, flow rate, pressure, and so forth are invariable or vary very slowly. In reality, the instability of the voltage for some reasons would cause fluctuation of rotational speed. In this process, the flow acceleration effect will play a very important role in performance parameters such as flow rate and head, which will be subjected to continuous and significant changes in a very short time. During the past 30 years, some investigations on transient performance of pumps have been carried out. For example, Tsukamoto and Ohashi first studied transient characteristics of centrifugal pump during starting period using performance experiment and theoretical calculation [1]. Subsequently, Thanapandi and Prasad used the method of characteristics to originally analyze the dynamic performances of a volute pump during normal startup and stopping periods [2]. Based on the internal flow equation of impeller and the one-dimensional motion equation of pipeline, Wang et al. numerically solved the external hydraulic performance of a mixed-flow pump during startup period [3]. Dazin et al. thought that the transient effect of turbomachinery depends not only on the acceleration rate and flow rate but also on velocity profiles and their evolution during all kinds of transient operating periods [4]. Zhang et al. first obtained the variation curves of rotational speed, flow rate, and head of a low-speed-specific centrifugal pump during starting period by experiment, and then, based on a transient generalized equation, the additional theoretical heads were quantificationally calculated and analyzed [5]. Wu et al. used experiment method to reveal transient effects of a closed-loop pipe system during all kinds of stopping periods [6], in which three inertia schemes of rotor in each stopping scheme were independently implemented to measure the torque, pressure, rotational speed, and so forth.

With the rapid development of computer technology, more and more scholars especially in China gradually used computational fluid dynamics (CFD) to research transient internal flow in turbomachinery. According to the geometric data of pump model in this existing paper, Li et al. designed 
TABLE 1: Main geometric parameters of pump model.

\begin{tabular}{lccc}
\hline Suction diameter $D_{i} / \mathrm{mm}$ & 50 & Inlet blade angle $\beta_{1} /\left(^{\circ}\right)$ & 25 \\
Discharge diameter $D_{o} / \mathrm{mm}$ & 40 & Outlet blade angle $\beta_{2} /\left(^{\circ}\right)$ & 25 \\
Blade number $Z$ & 5 & Impeller inlet width $b_{1} / \mathrm{mm}$ & 20 \\
Impeller diameter $D_{2} / \mathrm{mm}$ & 160 & Impeller outlet width $b_{2} / \mathrm{mm}$ & 10 \\
\hline
\end{tabular}

a new volute and built a closed-loop system to successfully carry out self-coupling startup simulation by means of given experimental result of rotational speed [7]. Except for the pure water medium, on the condition that the transmission medium is solid-liquid two-phase flow, the first author of this paper also successfully accomplished the numerical simulation of internal flow in centrifugal pump during rapid and slow startup periods [8]. Under the constant rotating speed, Wu et al. used CFD method to numerically simulate the transient internal flow in a centrifugal pump when the discharge valve is rapidly opened [9].

Tsukamoto et al. used experiments and singularity method to study the dynamic characteristics of centrifugal pumps subjected to fluctuating rotational speed [10]. It was found that the difference between dynamic and quasi-steady characteristics becomes more remarkable with the increased frequency of rotational speed fluctuations. Up to now, this paper is the only paper about fluctuating rotational speed of pump that we can find in published reports. Although Tsukamoto studied the transient behavior of pump by means of external performance, he did not reveal the variation characteristics of internal flow fields. In this paper, CFD method will be employed as an attempt to reveal more flow details and transient characteristics of a low-specific-speed centrifugal pump when its rotational speed is subjected to sinusoidal changes.

\section{Pump Model and Numerical Method}

2.1. Centrifugal Pump. The studied model is a prototype centrifugal pump with specific speed 45 . Its design parameters are as follows: flow rate is $6 \mathrm{~m}^{3} / \mathrm{h}$, head is $8 \mathrm{~m}$, and rotational speed $=1450 \mathrm{r} / \mathrm{min}$. The shape of the blades in impeller is twodimensional (2D) cylindrical profile and the volute is spiral. Its main geometric parameters can be found in Table 1; more detailed information about the pump can be seen in [8].

2.2. Computational Domain and Mesh. As known to all, the instantaneous rotational speed, flow rate, pressure, and so forth quickly vary with time when pump is subjected to fluctuating rotational speed. Therefore, it is very difficult to accurately specify the boundary conditions at the inlet and the outlet of the pump in the absence of experimental results. In this paper, a closed-loop pipe system including the centrifugal pump is built to accomplish self-coupling calculations so as to eliminate the difficulty of implementing boundary conditions in simulation. In this calculation, the boundary conditions at the inlet and the outlet of the pump are not required to be set after the variation of rotational speed is given.

The real pipe system containing valves, tanks, pipelines, and so forth can be simplified as calculation model. The dimensions of the tank are $500 \times 500 \times 1000 \mathrm{~mm}$ in Figure 1(a); more detailed geometric information about the simplified model can be seen in [8]. Regulating the simplified valve diameter can control the resistance of the whole pipe system. Under a certain rotational speed, reducing the valve diameter can increase local hydraulic loss and decrease the flow rate. In the present study, the valve diameter is $10 \mathrm{~mm}$. Under the rated rotational speed, the final flow rate is about $4.3337 \mathrm{~m}^{3} / \mathrm{h}$. The computational grids are generated using the commercial CFD software GAMBIT. The grid generated for the centrifugal pump is shown in Figure 1(b) in which the grids in impeller and volute are unstructured tetrahedron grids. The grid dependency study is carried out for the present model; it is found that the head correlation is less than $1 \%$ and there is almost no difference among the flow fields. Consequently, the influence of the grid numbers on the numerical results can be ignored. The final grid number used in the computation is $1,841,410$, where the grid numbers in impeller, volute, and tank are 616,018, 383,233, and 496,800, respectively. The value of $y+$ is taken as about 30 near the boundary wall. The grid number can be used to correctly predict the external performance and capture the macroscopical basic flow phenomenon.

2.3. Numerical Method. The commercial code FLUENT is used to calculate the transient flow inside the pump subjected to fluctuating rotational speed. On the top of the tank, the gage pressure is set as $0 \mathrm{~Pa}$ so as to be consistent with the real state. The variation rule of fluctuating rotational speed is written into FLUENT using user defined function (UDF). In this paper, the instantaneous rotational speed is given as the following function of time:

$$
n(t)=n_{D}+A \sin (B t),
$$

where $n_{D}$ is the design rotational speed. $A$ is the fluctuation amplitude of rotational speed and is 145 in the present study. Angular velocity $B=20 \pi$.

The dynamic slip region (DSR) method is used to solve the transient flow inside the pump subjected to fluctuating rotational speed $[7,8]$. The RNG $k-\varepsilon$ turbulence model including the influence of high strain rate and large curvature overflowing has been verified that it is suitable to simulate the flow inside a pump. No-slip boundary conditions are applied on all walls. The standard wall function is also used 


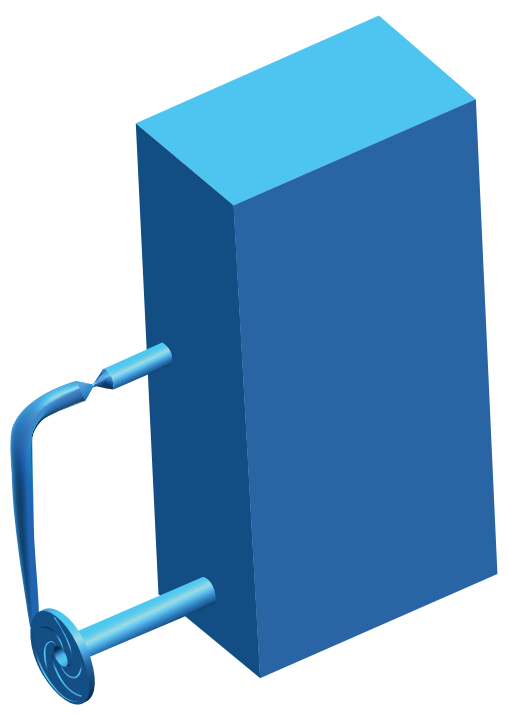

(a) Sketch of closed-loop pipe system

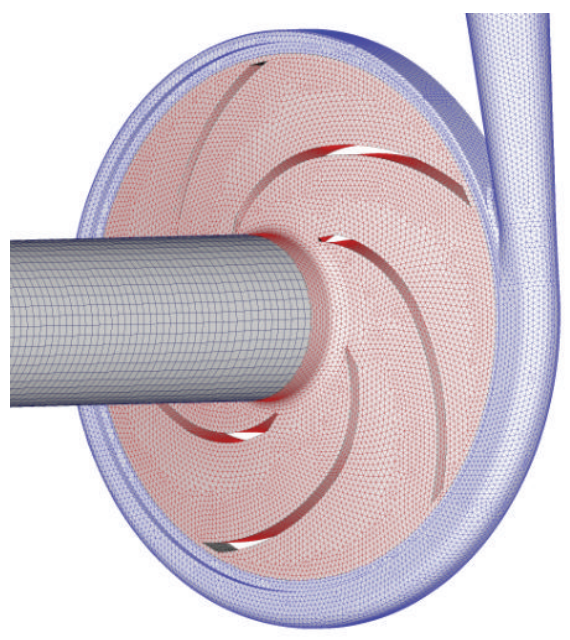

(b) Grids in centrifugal pump

FIgURE 1: Configuration of computational domain and grid generation.

to deal with the flow near the walls. The SIMPLE algorithm is used to solve the discretized equations, including velocity and pressure update to enforce mass conservation and eventually to obtain the pressure field. The second order upwind scheme is used to discretize the convective terms, and the central difference scheme is applied for the diffusion term. The time dependent term is in the first order implicit scheme. The residual tolerances are set as 0.0001 . The time step is $0.0001 \mathrm{~s}$ and the whole fluctuating time is $1 \mathrm{~s}$.

\section{Results and Discussions}

3.1. Pump Head and Flow Rate. At the transient operating conditions, the total instantaneous head consists of the steady head and the flow inertial head caused by flow acceleration effect [7]. Figure 2 shows the time histories of the instantaneous flow rate and pump head when the rotational speed is fluctuated according to the rule in (1). It is seen that there generally are similar variation tendencies in the head and the flow rate as the rotational speed; namely, both of them rise with the increasing rotational speed, otherwise down. This phenomenon can be easily understood from the dependence of them on the rotational speed. But, meanwhile, some slight differences are also seen from Figure 2. As a whole, the head's responsiveness to fluctuating rotational speed is relatively satisfied, and both of them display very good synchronization in time. For example, the head and the rotational speed rise to their maximums at $0.025 \mathrm{~s}$ in the first period. It is found that the rotor-stator interaction between dynamic impeller and static casing makes the head show periodic fluctuation characteristic. Due to flow acceleration effect, the flow rate slightly lags behind the rotational speed. For instance, the rotational speed rises to the maximum at $0.025 \mathrm{~s}$ in the first period, while the moment when the flow rate rises to its maximum is about $0.035 \mathrm{~s}$. Moreover, compared with the

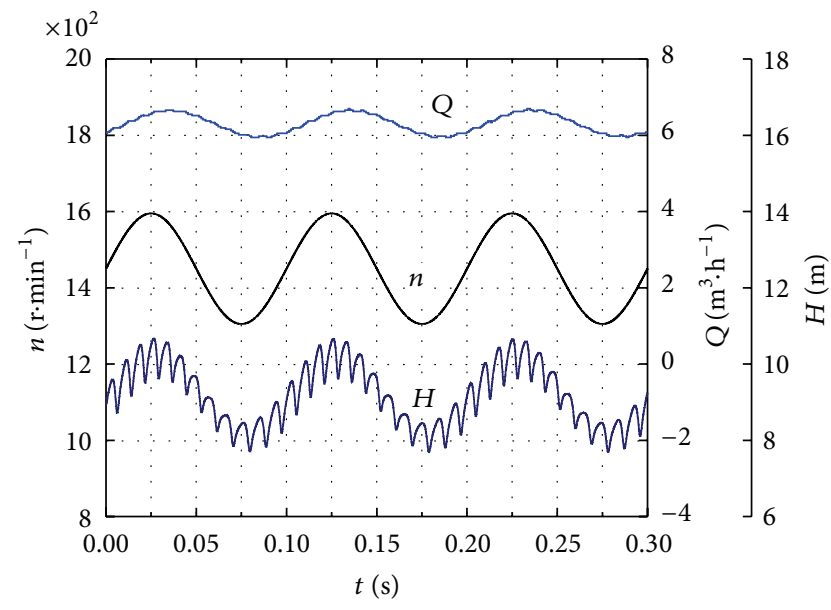

FIgURE 2: Time histories of head and flow rate of pump subjected to fluctuating rotational speed.

obvious fluctuation in the head, the fluctuation in the flow rate is very slight for rotor-stator interaction.

3.2. Nondimensional Head and Flow Rate. The fluctuating rotational speed causes every performance parameter of the pump to vary intensely. Therefore, its effects should be excluded from them so as to have a better understanding on the transient behavior. As such, the nondimensional flow rate coefficient and nondimensional head coefficient are defined as follows:

$$
\begin{gathered}
\phi(t)=\frac{Q(t)}{\pi D_{2} b_{2} u_{2}(t)}, \\
\psi(t)=\frac{2 \mathrm{~g} H(t)}{u_{2}^{2}(t)},
\end{gathered}
$$




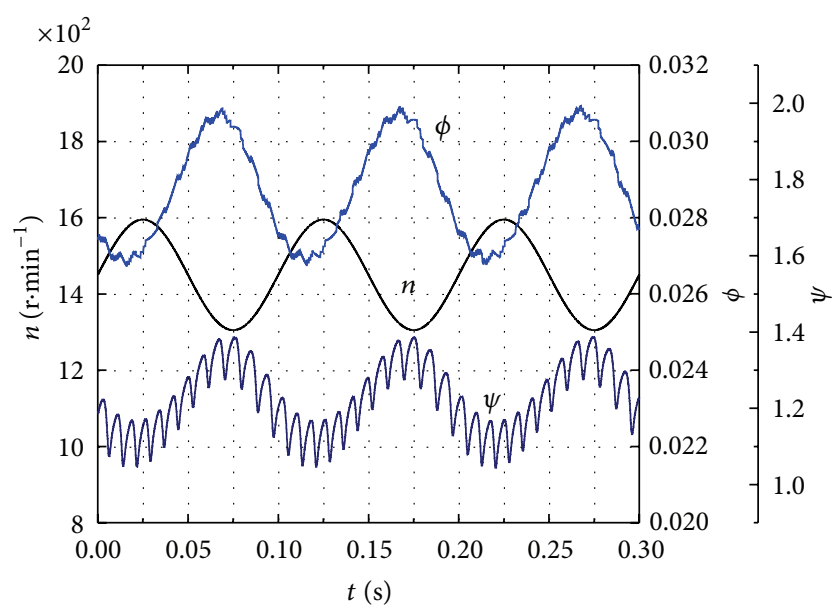

FIGURE 3: Time histories of nondimensional head and nondimensional flow rate.

where $u_{2}(t)$ is the impeller tip speed, $u_{2}(t)=\pi D_{2} n(t)$. Clearly, these two coefficients are independent of the rotational speed.

Figure 3 shows the time histories of the nondimensional flow rate coefficient and nondimensional head coefficient when the pump is subjected to fluctuating rotational speed. Clearly, the calculation results show that the nondimensional flow rate coefficient and nondimensional head coefficient are not constants, while being dependent on rotational speed. This result manifests that the similarity law of pumps or quasi-steady assumption is not suitable to assess the transient flow inside the pump. As a whole, the variation tendencies of these two coefficients are very similar but are opposite to that of the fluctuating rotational speed. When the rotational speed rises to the maximum, the nondimensional head coefficient decreases to the minimum, but the nondimensional flow rate coefficient is not the minimum. For example, the rotational speed rises to the maximum at $0.025 \mathrm{~s}$ in the first period, while the moment when the nondimensional flow rate coefficient reaches to its minimum is not $0.025 \mathrm{~s}$, but $0.015 \mathrm{~s}$. This result indicates that, in the whole process, the nondimensional flow rate coefficient is not synchronous with the rotational speed.

3.3. Static, Dynamic, and Total Pressures. In this study, the variation histories of the instantaneous static pressure, dynamic pressure, and total pressure at the inlet and the outlet of the centrifugal pump are also obtained through calculation, which can be seen in Figure 4 .

It can be clearly seen from Figure 4(a) that the variation trend of the static pressure at the inlet of the pump is different from that of the outlet. As a whole, the variation tendency of the static pressure at the outlet is the same as that of the rotational speed, and both of them increase or decrease to the maximum or the minimum; namely, the responsiveness to rotational speed is very perfect. However, for the static pressure at the inlet of the pump, its variation tendency is almost opposite to that of the rotational speed. Likewise, the rotor-stator interaction makes the static pressures show periodic fluctuation characteristics to some degree.
Figure 4(b) shows that the dynamic pressures at the inlet and the outlet of the pump slightly lag behind the rotational speed; this phenomenon is consistent with the variation result of the flow rate in Figure 2. This is because the dynamic pressure characteristic is directly determined by the flow rate characteristics. Moreover, it can be seen that the dynamic pressure at the outlet of the pump is higher than that at the inlet. In the present pump model, the discharge diameter of the pump is less than the suction diameter, and the former and the latter are $50 \mathrm{~mm}$ and $40 \mathrm{~mm}$, respectively. As such, the average velocity at the outlet is higher than that at the inlet; therefore, the dynamic pressure at the outlet is always higher than that at the inlet theoretically. It is also seen from Figure 4(b) that the magnitude of the dynamic pressures is very small due to the low flow rate in the present pump model. Generally speaking, the dynamic pressures' responsiveness to fluctuating rotational speed is relatively good.

As is well known, the total pressure is the sum of the static pressure and the dynamic pressure. Consequently, the former should be determined together by the latter two in theory. It is seen from Figures 4(a) and 4(b) that, at the outlet of the pump, the static pressure is much higher than the dynamic pressure, and both of them have the same variation tendency. Therefore, the total pressure profile in Figure 4(c) is almost the same as that of the static pressure. Likewise, the total pressure profile at the inlet is similar to that of the static pressure because the magnitude of the static pressure is relatively high comparing with the dynamic pressure. Note that the total pressure at the outlet of the pump is much higher than that at the inlet. As such, the total pressure characteristics at the outlet of pump would mainly determine the pump head characteristics. It is easy to see that both of them show the same evolution tendencies.

3.4. Impeller Shaft Power and Dynamic Reaction Force. Figure 5 shows the variation characteristics of the impeller shaft power and the impeller dynamic reaction force of the pump model when its rotational speed fluctuates according to the sinusoidal function, in which the definition of the impeller shaft power is written as

$$
P(t)=\frac{2 \pi M(t) n(t)}{60},
$$

where $M(t)$ is the instantaneous torque on impeller. In Figure 5, it is seen that the dynamic reaction force and the impeller shaft power show the same evolution tendencies; both of them synchronously increase or decrease with time. Meanwhile, it is also seen that both of them are ahead of the rotational speed; this can be attributed to the flow acceleration inertia. What is more, the rotor-stator interaction causes the impeller shaft power and the dynamic reaction force to display obvious fluctuation characteristics.

3.5. Flow Fields. Turbulence energy stands for the magnitude of turbulence pulsation, and thus its intensity and spatial distribution characteristics also reflect the magnitude of the viscous dissipation loss to a certain extent. In order to further understand the transient flow characteristics inside the pump 


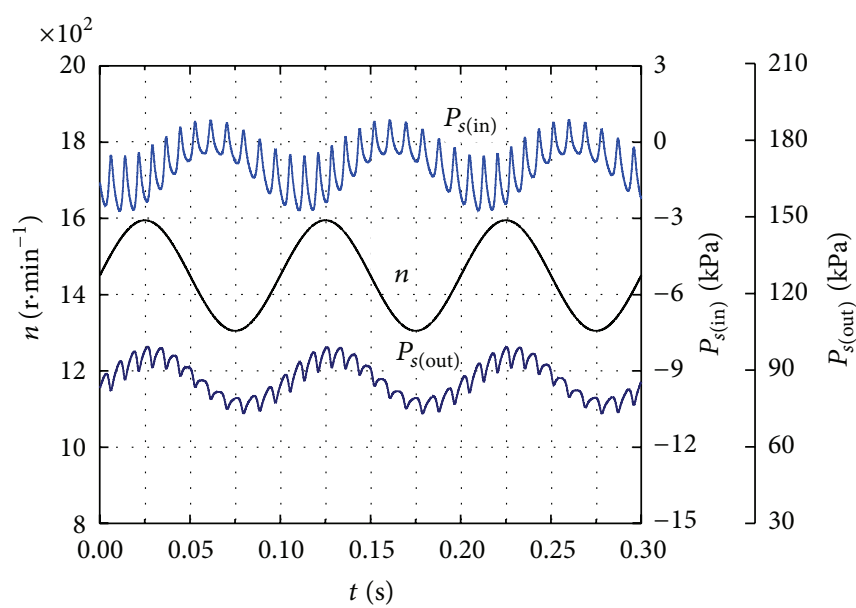

(a) Static pressure

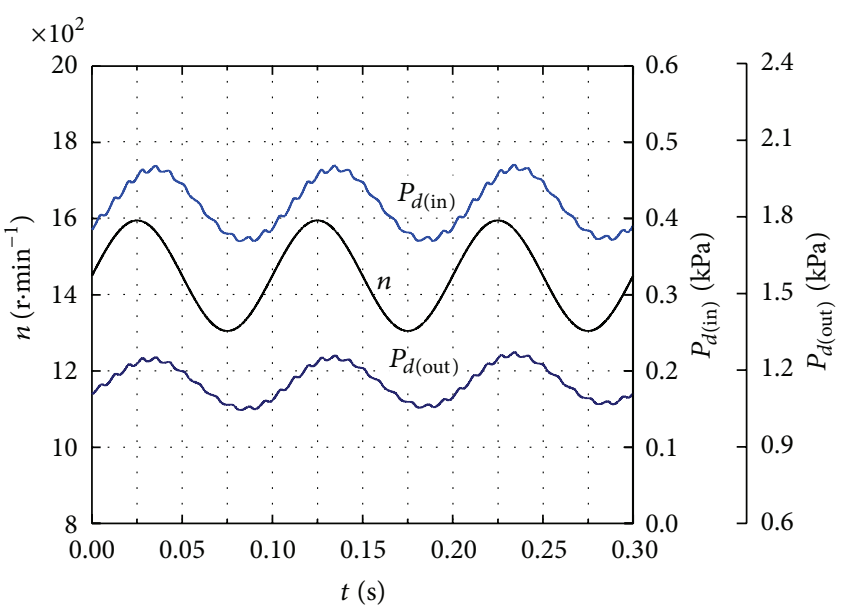

(b) Dynamic pressure

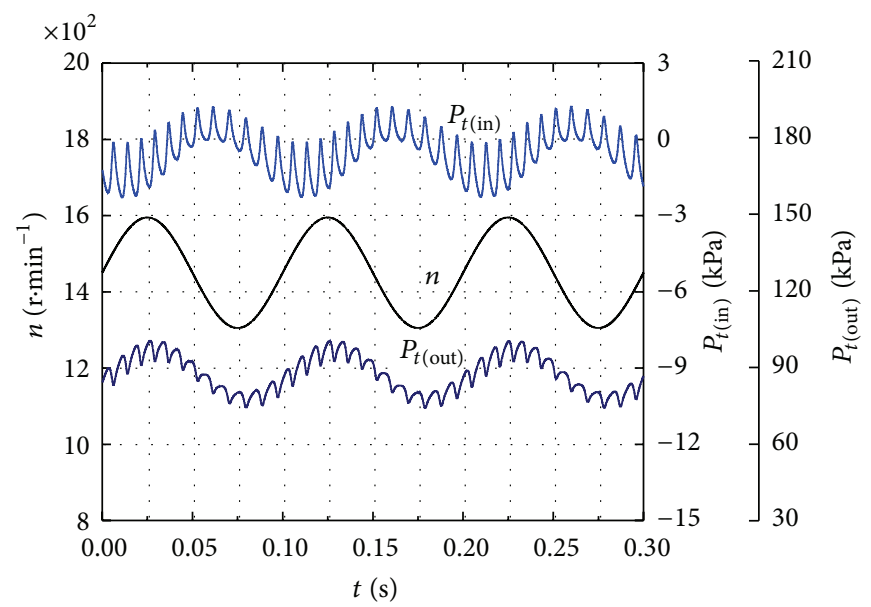

(c) Total pressure

FIGURE 4: Variations of pressures at inlet and outlet of pump subjected to fluctuating rotational speed.

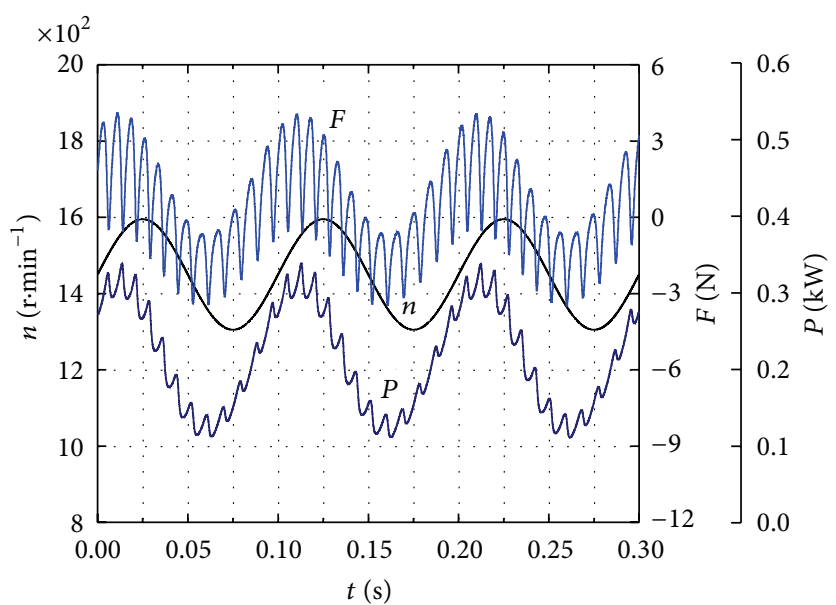

FIGURE 5: Characteristics of impeller shaft power and dynamic reaction force.

when it is subjected to fluctuating rotational speed, in this paper, the quasi-steady flow calculations for five rotational speeds are also carried out. Figures 6 and 7 show the evolution results of the relative streamlines and the turbulence energy in the transient and quasi-steady calculations. And in quasisteady calculations, the rotational speed is taken from the corresponding value at the same moment in the transient flow calculation. 


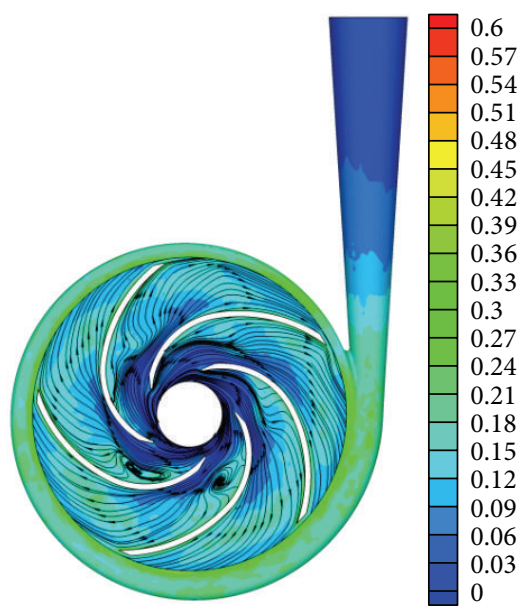

(a) $1312.1 \mathrm{r} / \mathrm{min}$

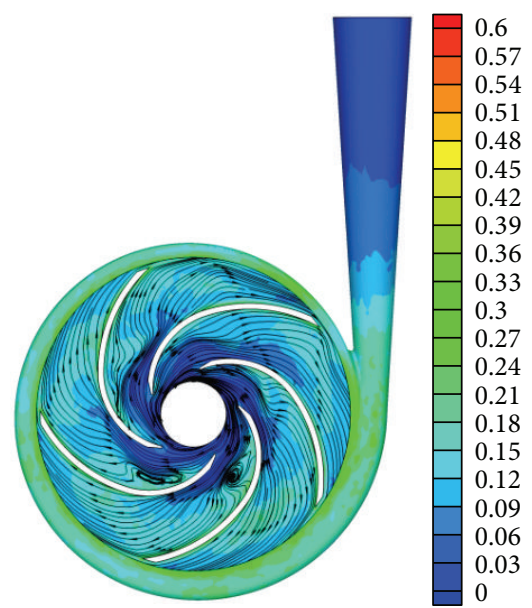

(b) $1364.8 \mathrm{r} / \mathrm{min}$

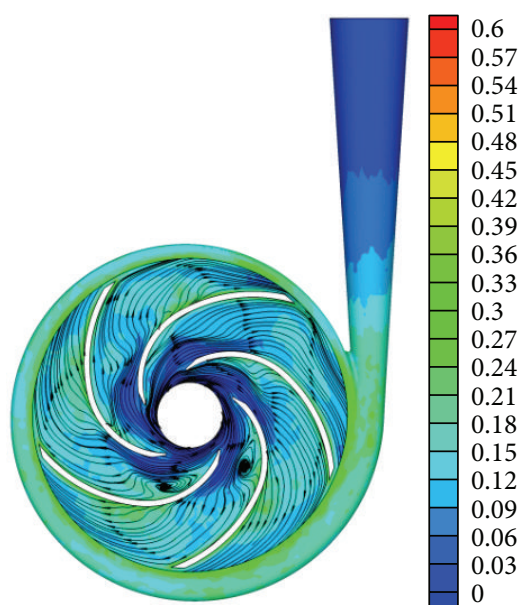

(c) $1450 \mathrm{r} / \mathrm{min}$

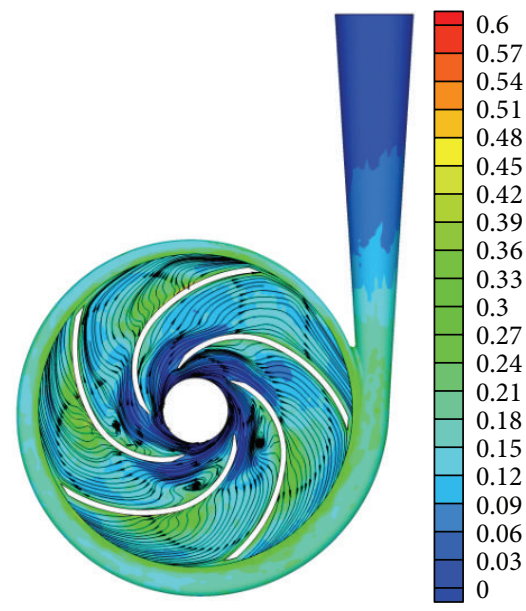

(d) $1535.2 \mathrm{r} / \mathrm{min}$

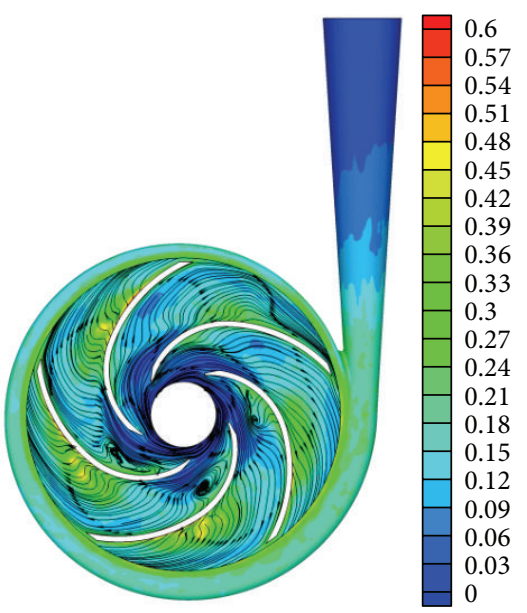

(e) $1587.9 \mathrm{r} / \mathrm{min}$

FIGURE 6: Evolutions of relative streamlines and turbulence energy $\left(\mathrm{m}^{2} \cdot \mathrm{s}^{-2}\right)$ in transient calculations.

It can be clearly seen that, in the transient flow calculation, the distribution of the turbulence energy is relatively uniform; namely, the intensity of the turbulence energy in each impeller channel is almost identical. However, in the quasisteady flow calculations, the distribution difference in each impeller channel is very obvious. It is easy to see that, in this impeller channel which is always closed to the volute tongue, the intensity of the turbulence energy is always the minimum compared with that in other channels. That can be attributed to the fact that the volute tongue structure plays a dominant role in the internal flow inside the pump especially for the impeller channel closing the volute tongue. Moreover, it looks like that the intensity of the turbulence energy in quasi-steady flow calculations is weaker than that in transient flow calculation as a whole. That is because the flow acceleration in the quasi-steady flow calculations is relatively smaller compared with that in the transient flow calculation. The smaller flow acceleration will result in the stronger turbulence pulsation, and thus the turbulence energy also becomes larger.

\section{Conclusions}

Based on the given fluctuating rotational speed, a closedloop pipe system including pump model is established to accomplish unsteady flow calculations inside a centrifugal pump subjected to fluctuating rotational speed. The calculation results not only contain the external performance but also contain the internal flow fields. The result shows that the head's responsiveness to fluctuating rotational speed is very good, while the flow rate's responsiveness is delayed. The nondimensional flow rate and head coefficients are dependent on the rotational speed, and the variation tendencies of them are opposite to that of the fluctuating rotational speed as a whole. The similarity law and the quasi-steady assumption are not suitable to accurately assess the transient characteristics in transient operation conditions. The variation characteristics of the static pressure at the inlet and the outlet of the pump are almost completely opposite, wherein the static pressure characteristics at the outlet are similar to those of the rotational speed. In the quasi-steady flow 


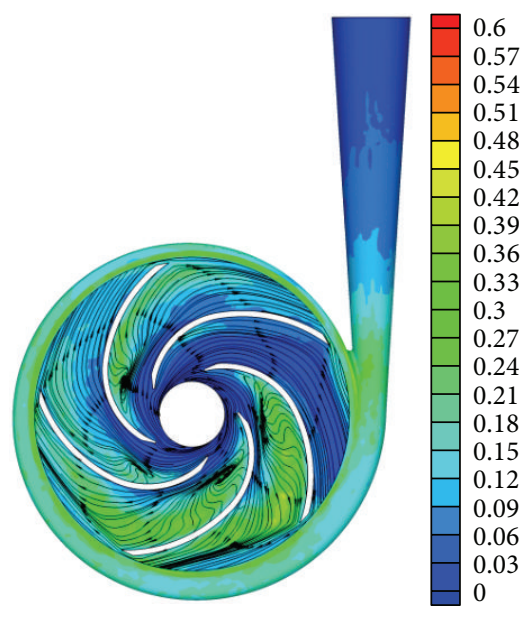

(a) $1312.1 \mathrm{r} / \mathrm{min}$

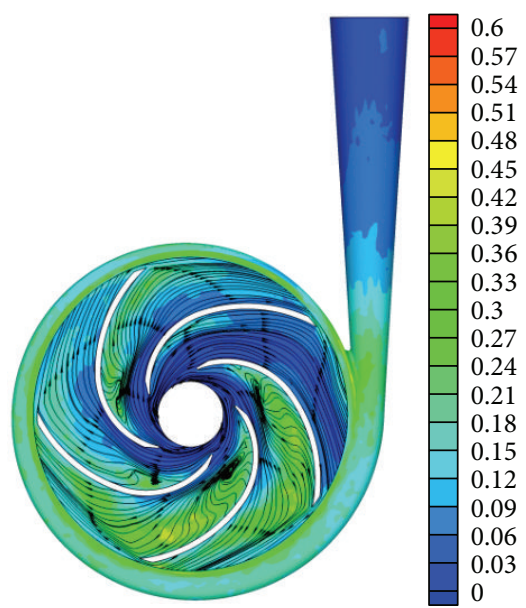

(b) $1364.8 \mathrm{r} / \mathrm{min}$

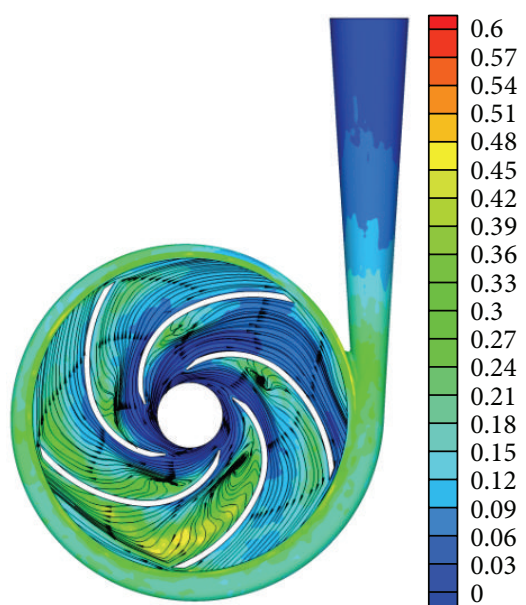

(c) $1450 \mathrm{r} / \mathrm{min}$

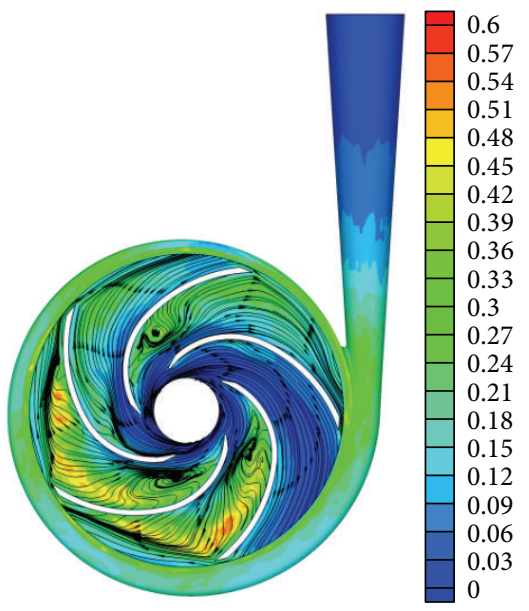

(d) $1535.2 \mathrm{r} / \mathrm{min}$

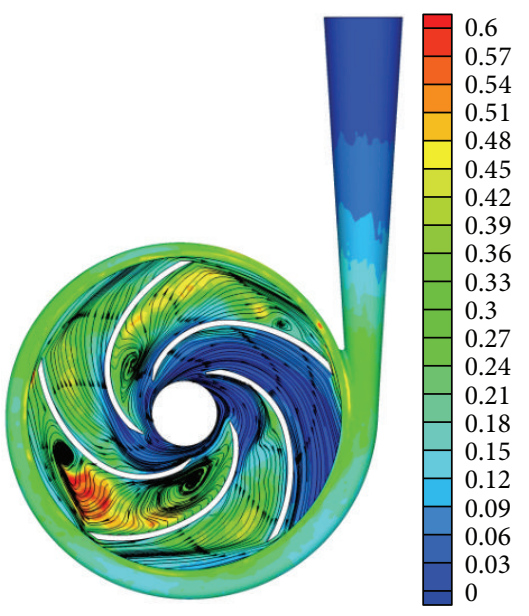

(e) $1587.9 \mathrm{r} / \mathrm{min}$

FIGURE 7: Evolutions of relative streamlines and turbulence energy $\left(\mathrm{m}^{2} \cdot \mathrm{s}^{-2}\right)$ in quasi-steady calculations.

calculation, the intensity of the turbulence energy becomes weaker in this channel that is closed to the volute tongue.

\section{Conflict of Interests}

The authors declare that there is no conflict of interests regarding the publication of this paper.

\section{Acknowledgments}

This work was supported by the National Natural Science Foundation of China (no. 51276172) and Zhejiang Provincial Natural Science Foundation of China (no. LY14E090011).

\section{References}

[1] H. Tsukamoto and H. Ohashi, "Transient characteristics of a centrifugal pump during startup period," ASME Journal of Fluids Engineering, vol. 104, no. 1, pp. 6-13, 1982.

[2] P. Thanapandi and R. Prasad, "Centrifugal pump transient characteristics and analysis using the method of characteristics,"
International Journal of Mechanical Sciences, vol. 37, no. 1, pp. 77-89, 1995.

[3] L. Q. Wang, D. Z. Wu, S. Y. Zheng, and Z. Hu, "Study on transient hydrodynamic performance of mixed-flow-pump during starting period," Journal of Zhejiang University (Engineering Science), vol. 38, no. 6, pp. 751-755, 2004.

[4] A. Dazin, G. Caignaert, and G. Bois, "Transient behavior of turbomachineries: applications to radial flow pump startups," Journal of Fluids Engineering-Transactions of the ASME, vol. 129, no. 11, pp. 1436-1444, 2007.

[5] Y. L. Zhang, Z. C. Zhu, H. S. Dou et al., "Experimental and theoretical study of a prototype centrifugal pump during startup period," International Journal of Turbo \& Jet-Engines, vol. 30, no. 2, pp. 173-177, 2013.

[6] D. Z. Wu, P. Wu, and S. Yang, "Transient characterisitcs of a close-loop pipe system during pump stopping periods," ASME Journal of Pressure Vessel Technology, vol. 136, no. 2, Article ID 021301, 2014.

[7] Z. Li, D. Wu, L. Wang, and B. Huang, "Numerical simulation of the transient flow in a centrifugal pump during starting period," Journal of Fluids Engineering-Transactions of the ASME, vol. 132, no. 8, Article ID 081102, 8 pages, 2010. 
[8] Y. L. Zhang, Transient internal flow and performance of centrifugal pumps during startup period [Ph.D. thesis], Zhejiang University, Hangzhou, China, 2013.

[9] D. Wu, P. Wu, Z. Li, and L. Wang, "The transient flow in a centrifugal pump during the discharge valve rapid opening process," Nuclear Engineering and Design, vol. 240, no. 12, pp. 40614068, 2010.

[10] H. Tsukamoto, H. Yoneda, and K. Sagara, "Response of a centrifugal pump to fluctuating rotational speed," ASME Journal of Fluids Engineering, vol. 117, no. 3, pp. 479-484, 1995. 


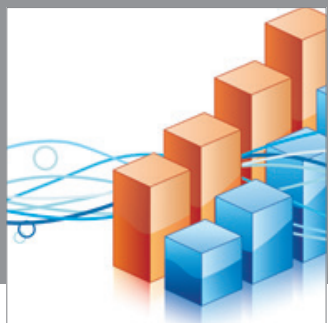

Advances in

Operations Research

mansans

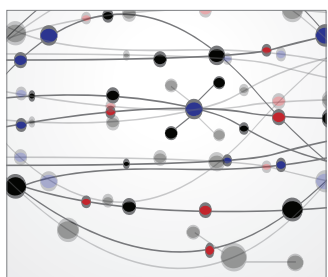

The Scientific World Journal
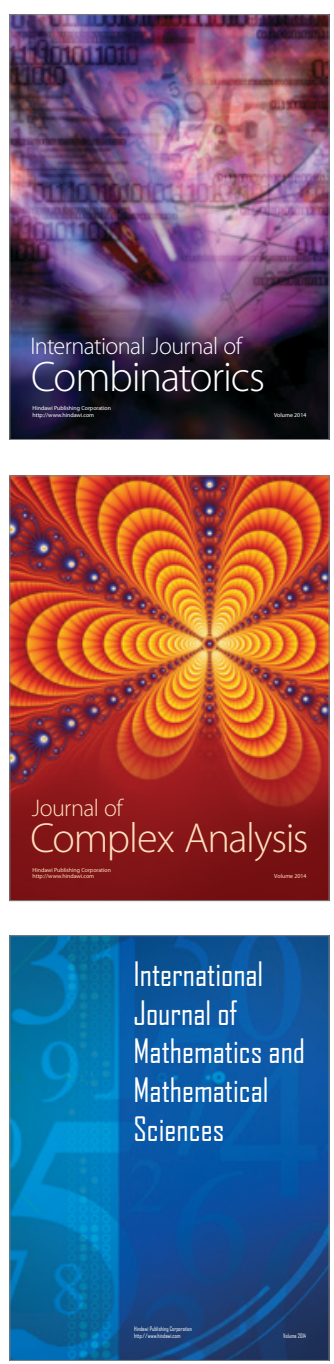
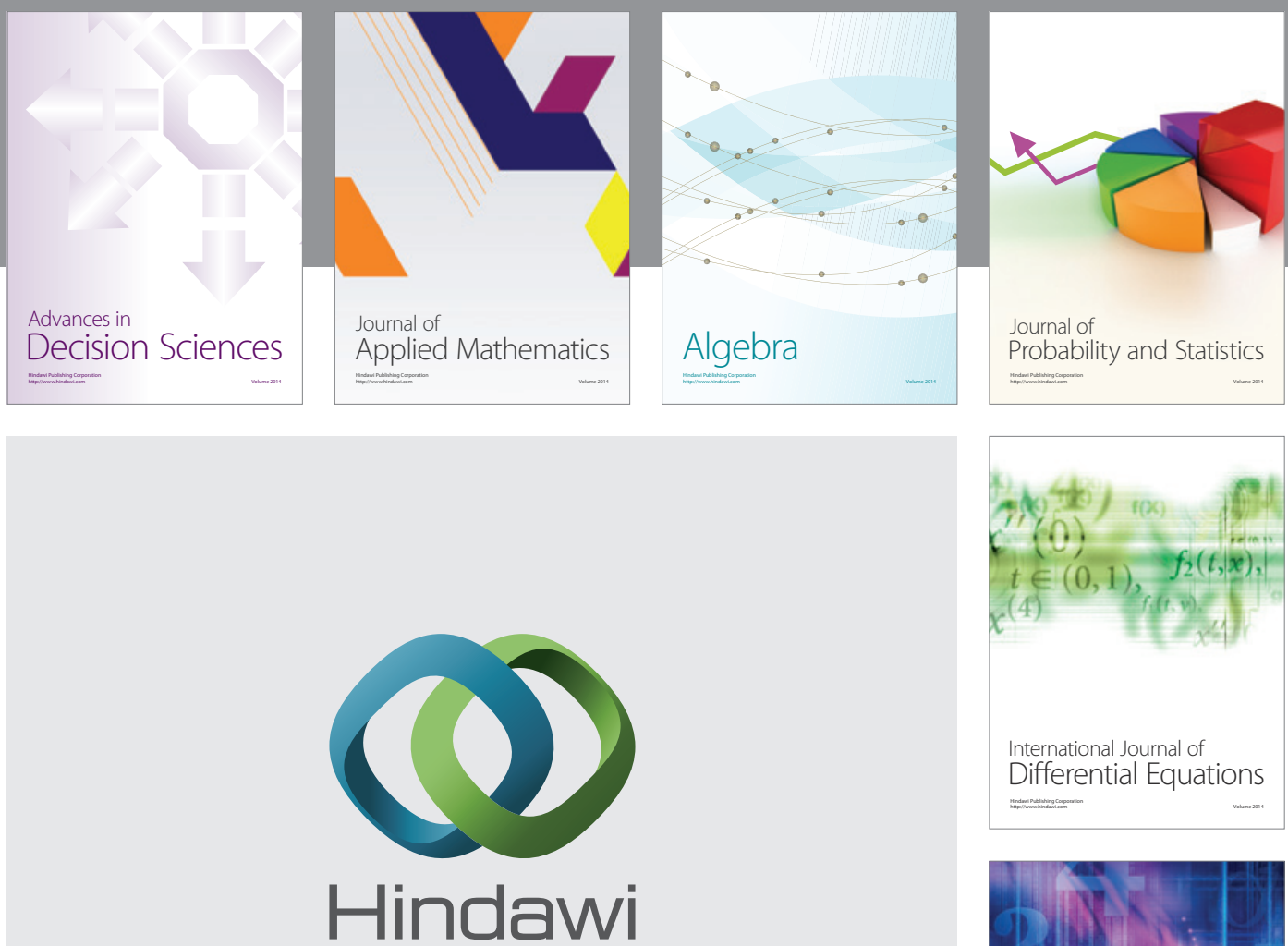

Submit your manuscripts at http://www.hindawi.com
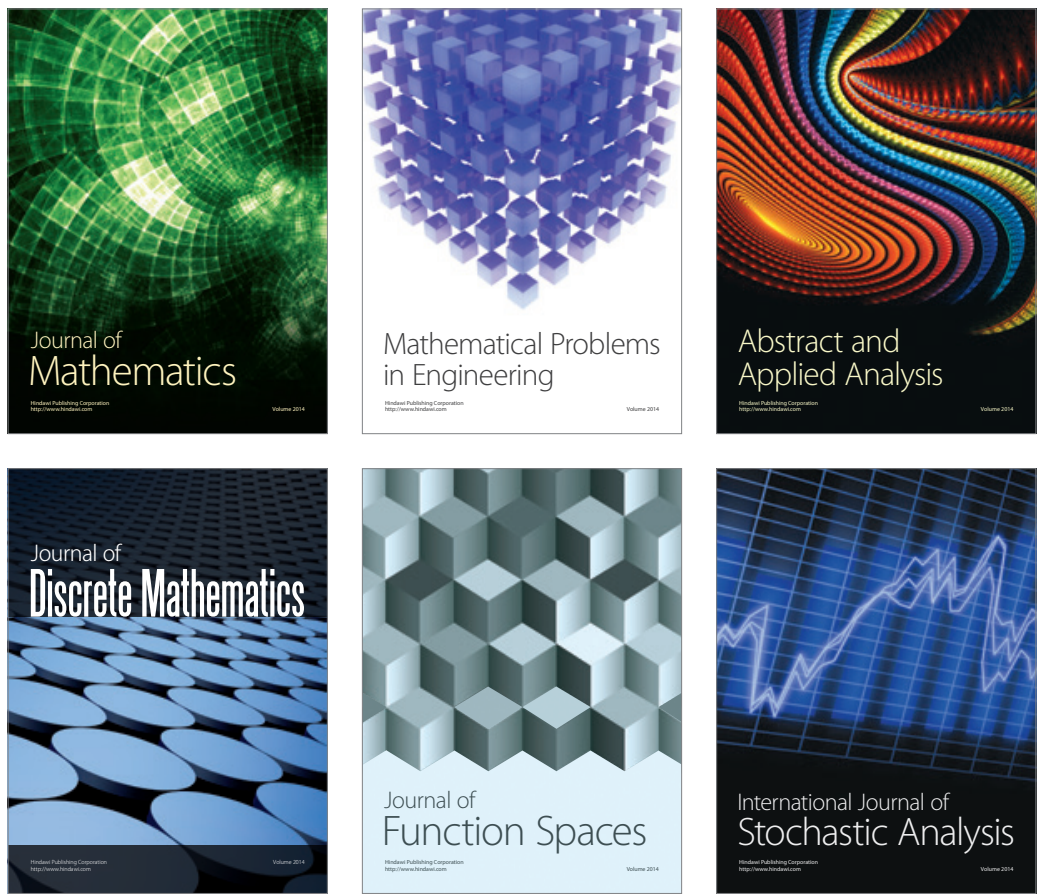

Journal of

Function Spaces

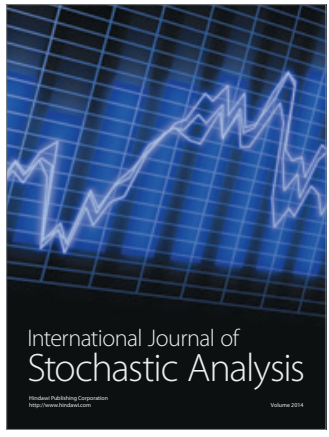

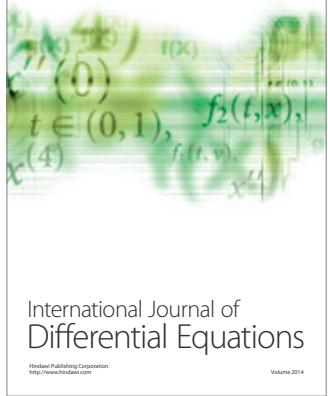
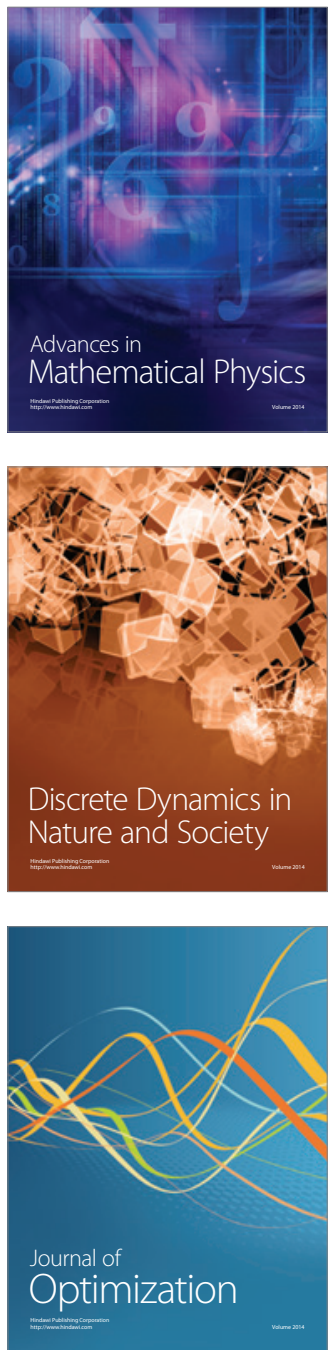